EPiC Series in Health Sciences
Volume 3, 2019, Pages 334-337
$\begin{aligned} & \text { CAOS 2019. The 19th Annual Meeting of the International } \\ & \text { Society for Computer Assisted Orthopaedic Surgery }\end{aligned}$

\title{
Development of a Biofeedback Wearable Sensor System to Improve Rehabilitation Following Surgical Repair of Achilles Tendon Tears
}

\author{
Sukhi Singh ${ }^{1}$, Tim Lee ${ }^{1}$, and Joshua W Giles ${ }^{1}$ \\ ${ }^{1}$ Orthopaedic Technologies and Biomechanics Lab, Department of Mechanical Engineering, \\ University of Victoria, Victoria, Canada \\ jwgiles@uvic.ca
}

\begin{abstract}
This paper describes the development, functionality, and initial testing of a wearable sensor system and companion smartphone app intended to support the rehabilitation of Achilles injury patients by providing 1 ) real time biofeedback, which can help patients adhere to rehabilitation restrictions; 2 ) exercise support to encourage patients to correctly perform all rehabilitation activities; 3 ) data summaries to clinicians in order to allow appropriate interventions when necessary. The wearable system is composed of insole pressure sensors, a calf muscle activation sensor, and inertial measurement units, whose data are communicated to the smartphone app via a Bluetooth enabled microcontroller. Initial testing demonstrated the quality of the data recorded by the sensors and the ability of those data to be used to identify functional activities like walking and stairclimbing.
\end{abstract}

\section{Introduction}

Achilles tendon rupture is a common traumatic injury with an incidence of 18.0 to 29.3 per 100,000 person-years (Sheth et al.). Surgery followed by an extended rehabilitation protocol is the preferred treatment for large/complete ruptures. Rehabilitation often lasts $>10$ weeks and involves at least four stages with variable constraints on patient activity and rehab exercises (Kearney et al.). As a result of this complexity, it is often difficult for patients to assess if they are properly adhering to the protocol's guidelines and correctly performing exercises both of which put patients at risk for re-injury (Costa et al.). Furthermore, there remains controversy regarding the optimal rehabilitation protocol as it is currently impossible for clinicians to correlate the quality of patient outcomes with their adherence to the protocol (Kearney and Costa). Therefore, our goal was to develop a coupled wearable sensor system and smartphone app for ankle injury rehab that could provide real-time biofeedback, rehab exercise support, and produce overall statistics for clinician review. 


\section{Materials \& Methods}

Device Development: To achieve these goals, this system was developed with three modules: 1) wearable sensors, 2) a Bluetooth enabled microcontroller, and 3) a smartphone app. The physical device was designed to be comfortably worn both when patients are confined to an Aircast and while wearing normal footwear.

Given the goal of providing biofeedback to improve protocol adherence, it was necessary to choose or develop sensors that could assess the degree of tendon loading - the root cause of post-operative repair failure - and/or provide biomechanical data indicative of prohibited functional activities (e.g. stair climbing). To this end, we developed an integrated device that yields calf muscle activation data, shank-foot kinematics, and multi-sector foot pressure values (Figure 1). It was believed that these multimodal data would be sufficient to enable the identification of any relevant functional activities. After evaluating multiple options, including traditional electromyography (EMG), the current system was designed with a custom muscle activation sensor based on calf volume change as this is more appropriate than EMG for persistent use. Shank-foot kinematics are determined using two 9 axis IMUs (MPU 6050, Invensense, USA) one in the shank mounted muscle sensor/microcontroller housing and the other in the insole component. Finally, foot pressure is evaluated using Force Sensitive Resistors (FSRs) located in the heel and ball of the insole. The two components are linked by a USB-C cable.
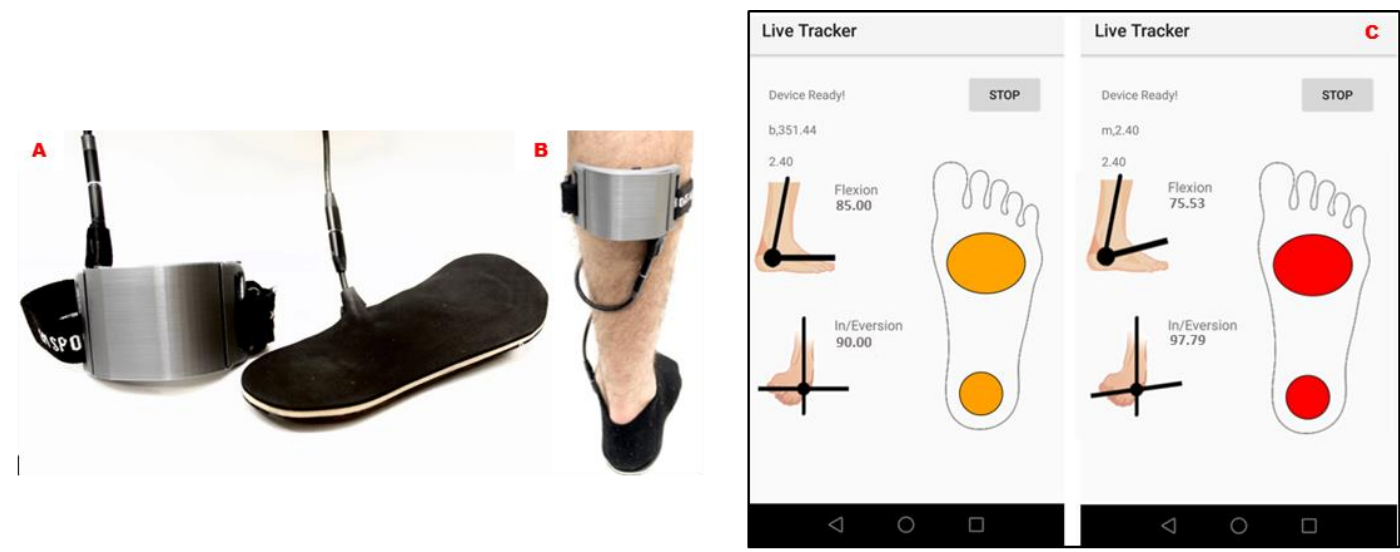

Figure 1: A) Shank and insole modules of biofeedback system connected by USB-C; B) the system being worn by a user; C) smartphone app "Exercise Mode" display. Two bars on left side represent the relative shank-foot orientation measured by the IMUs in the calf strap and insole. The colours inside the foot graphic indicate the degree of weight bearing, with yellow indicating a minimal amount, and red indicating fully body weight.

These sensors are sampled by the Bluetooth enabled microcontroller (Bluefruit Featherboard M0, Adafruit, USA) that communicates the raw data to the smartphone app, while also conducting event identification (i.e. functional activities), logging the raw data to an embedded micro-SD card, and providing haptic feedback using a vibration motor if a critical event is identified (e.g. calf muscle overloading). The event identification capability, which is currently being refined, is based on machine learning of the sensor data during activities that violate the rehab protocol guidance.

Smart Phone App: The smartphone app has been developed with four functions: 1) continuous data visualization, 2) real-time biofeedback, 3) rehabilitation exercise mode, and 4) long-term data logging. The first two functions have been implemented while the latter two are under development. The purpose of the first function is to enable patients to visualize their functional data, which will help them to better understand the clinical guidelines. The second function acts to provide real-time biofeedback by way of 'push' notifications (i.e. phone vibration and/or audible notification) when 
patients are identified to be exceeding acceptable functional limits. The third function is intended to help patients correctly perform rehab exercises and to illustrate their functional progress (or lack thereof) as a means of encouraging adherence to the exercise regime. Finally, the logging function will provide a compact data representation that clinicians can easily review and use to influence future rehabilitation protocols.

Validation: Preliminary test results have been recorded to validate the system's ability to quantify muscle activity, ankle rotation, and insole loading. The muscle activation FSR response was assessed for a voluntary calf activation activity. The ability to accurately measure shank-foot relative angles was assessed by having a user flex, extend, invert, and evert their ankle to multiple positions which were simultaneously measured using the IMUs and a goniometer. To assess the pressure pads installed into the insole, known loads were applied to the heel and toe sensors independently and repeatedly, with analog voltage results represented in 10 bit form.

\section{Results}

The muscle activation FSR's data was sensitive to positioning but - when positioned $\sim 4$ inches inferior to the popliteal fossa and rotated to align with the medial gastrocnemius edge - produced gastrocnemius activation data in agreement with Baskin \& Paolini (1966) who found muscle volume will exhibit a "rapid increase, a subsequent decrease, and a return to the initial volume" (Figure 2A).

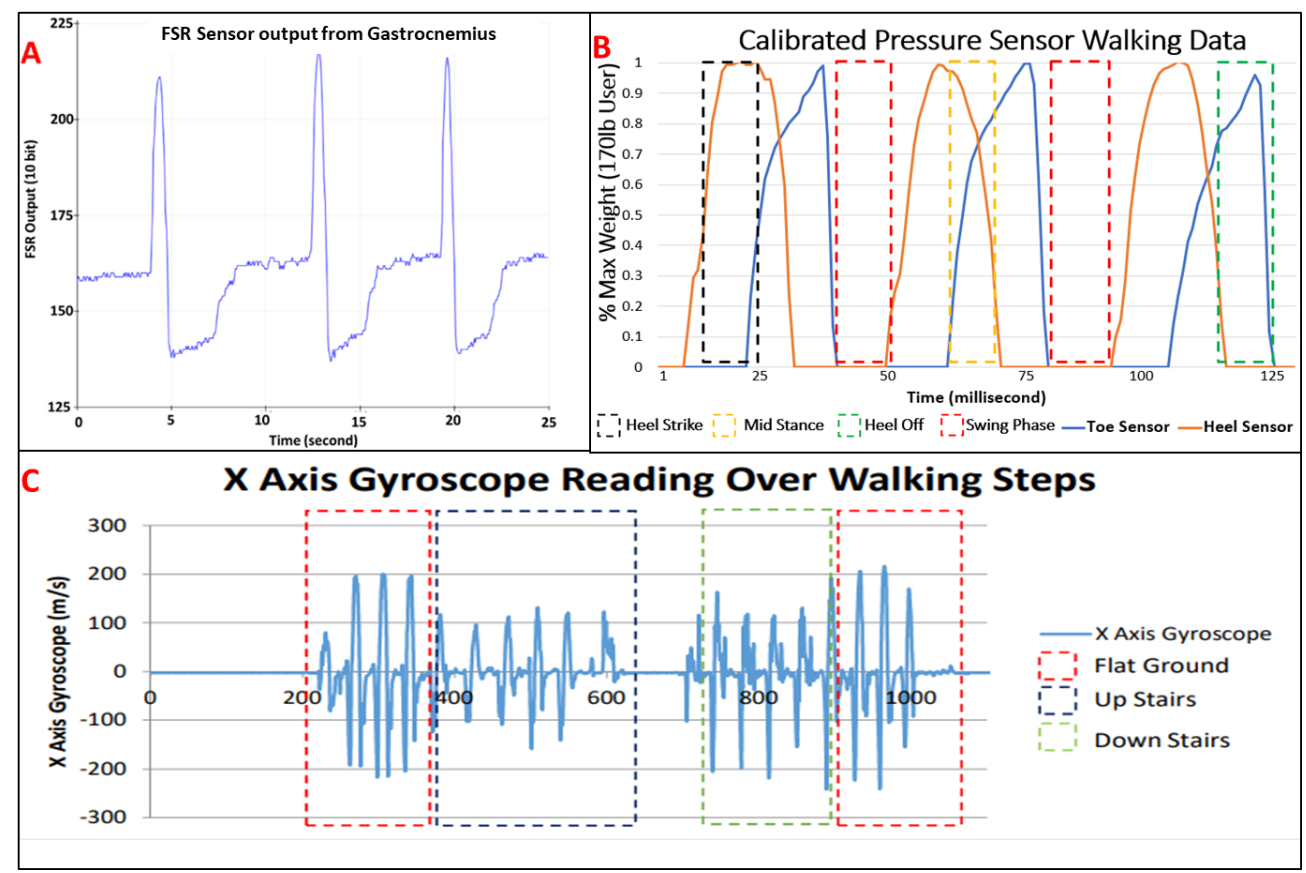

Figure 2: A) Analog output from FSR sensor as a user performs voluntary contractions of the gastrocnemius. B) Data for each phase of gait can be extracted from the full waveform to analyze the user's ankle weight bearing biomechanics during walking. C) X-axis gyroscope data, which is directed medio-laterally, outlining the changes in the angular velocity of the shank during stair climbing and walking on flat ground.

The insole sensors are successfully able to distinguish between different gait parameters by detecting the changes in load on the forefoot and hindfoot as shown in Figure 2B. 
The IMU sensors demonstrated how angular displacement is different when a user is stair climbing or walking on flat ground. Additionally, other parameters such as linear acceleration, muscle activity of the gastrocnemius, and insole pressure sensor values also assist in the event identification algorithms.

\section{Discussion}

Wearable sensor systems are an increasingly important part of providing computer assistance to orthopaedic surgery. To date these systems have focused on clinical follow up after joint replacement, most notably knee replacement (e.g. Claris Healthcare's Relfex system)(RebalanceMD, 2018). These systems have begun to show signs of success but remain very limited in the types of data they measure and the functionality they provide to patients and clinicians alike. The system described here is novel with respect to its application to the ankle, the multi-modal data it employs, and its broad functionality. Preliminary tests of this system show promise with respect to its ability to provide useful biofeedback data. It is our belief that this system will improve patient adherence and thus functional outcomes, limit patient re-injury by identifying, in real-time, restricted functional activities, and provide clinicians with objective data to improve their rehabilitation protocols.

\section{References}

Costa, M. L., et al. "Randomised Controlled Trials of Immediate Weight-Bearing Mobilisation for Rupture of the Tendo Achillis." The Journal of Bone and Joint Surgery. British Volume, vol. 88-B, no. 1, The British Editorial Society of Bone and Joint Surgery, Jan. 2006, pp. 69-77, doi:10.1302/0301620X.88B1.16549.

Kearney, R. S., and M. L. Costa. "Current Concepts in the Rehabilitation of an Acute Rupture of the Tendo Achillis." The Bone \& Joint Journal, vol. 94-B, no. 1, 2012, pp. 28-31, doi:10.1302/0301620X.94B1.28008.

Kearney, Rebecca S., et al. "A Systematic Review of Early Rehabilitation Methods Following a Rupture of the Achilles Tendon." Physiotherapy, vol. 98, no. 1, Mar. 2012, pp. 24-32, doi:10.1016/j.physio.2011.04.349.

"MPU-6050|TDK", Invensense.com. [Online]. Available:

https://www.invensense.com/products/motion-tracking/6-axis/mpu-6050/. [Accessed: 12- Jan- 2019].

"Overview | Adafruit Feather M0 Bluefruit LE | Adafruit Learning System", Learn.adafruit.com. [Online]. Available: https://learn.adafruit.com/adafruit-feather-m0-bluefruit-le/overview. [Accessed: 12- Jan- 2019].

R. Baskin and P. Paolini, "Muscle Volume Changes," Journal of General Physiology, vol. 49, no. 3, pp. 387-404, 1966.

RebalanceMD. New Monitoring Technology Reduces TKA Post-Acute Care Costs and Improves Patient Satisfaction The Study. 2018, www.clarisreflex.com.

Sheth, U., et al. "The Epidemiology and Trends in Management of Acute Achilles Tendon Ruptures in Ontario, Canada: A Population-Based Study of 27607 Patients." The Bone \& Joint Journal, vol. 99B, no. 1, Jan. 2017, pp. 78-86, doi:10.1302/0301-620X.99B1.BJJ-2016-0434.R1. 